\title{
Analyse Economique De La Restauration Des Aires Protegees En Côte D'ivoire : Cas Du Parc National De Taï
}

\author{
Moussa Sangare \\ Université Alassane OUATTARA de Bouaké (Côte d'Ivoire)
}

doi: 10.19044/esj.2017.v13n1p182 $\quad$ URL:http://dx.doi.org/10.19044/esj.2017.v13n1p182

\begin{abstract}
This paper analyses the optimal conditions of the imperative restoration of the National Park of Tai. Particularly, it aims to study, using the contingent valuation method, the feasibility of a voluntary retrocession of agricultural parcels located in the vicinity of this park. Thus a simple censored tobit model is used to identify the determinants of willingness to accept of smallholders. Data collection was conducted among 508 households settled in the neighboring localities of this park.

Statistical analysis of survey data reveals initially the unpopularity and difficulties of implementing such a measure given the low commitment of smallholders to the proposed policy.

Secondly, econometric analysis identifies the instruction attainment, part-time practice of farming, membership in some support group, previous farming experience, the extend of ceded parcel, farm-home distance and environmental sensitivity of the individual like the main explicative variables of the willingness to accept of smallholders.
\end{abstract}

Keywords: Conservation of forest resources, Contingent valuation method, National Park of Taï

\section{Resume}

Cette étude analyse les conditions optimales de l'impérieuse restauration du Parc National de Taï. Elle ambitionne notamment d'étudier, à partir de la méthode d'évaluation contingente, la faisabilité d'une rétrocession volontaire des parcelles agricoles situées dans l'aire d'adhésion de cet îlot forestier. Ainsi, un modèle tobit censuré simple est utilisé pour identifier les déterminants du consentement à recevoir des petits fermiers. Une collecte de données a été réalisée auprès de 508 ménages résidant dans les localités limitrophes. 
L'analyse statistique de ces données d'enquête révèle dans un premier temps l'impopularité et les difficultés de mise en œuvre d'une telle politique compte tenu de la faible adhésion des paysans à celle-ci. Dans un deuxième temps, l'analyse économétrique identifie le niveau d'instruction, l'exercice à temps partiel de l'activité agricole, l'appartenance à un groupe d'entraide, l'exercice d'un emploi précédemment à l'agriculture, les superficies cédées, la distance séparant l'exploitation du lieu d'habitation et la sensibilité environnementale de l'individu comme les principales variables explicatives du consentement à recevoir des paysans.

Mots-clés : Préservation des ressources forestières, Méthode d'évaluation contingente, Parc National de Taï

\section{Introduction}

Protéger les ressources forestières est devenue une priorité absolue pour les autorités ivoiriennes. Ces dernières traduisent leur volonté par la mise sous tutelle de plusieurs massifs forestiers sur toute l'étendue du territoire. Cette décision vise à constituer des réservoirs naturels et à freiner le "déclin de la biodiversité" dû en grande partie à la réduction drastique des superficies forestières (Konaté et Kampmann, 2010 ; Brou et al., 2005).

Ainsi, les autorités ivoiriennes ont procédé, par la promulgation du Décret $n^{\circ}$ 77-348 du 03 Juin 1977, à l'érection de 350000 hectares de forêt en aire protégée qu'elles ont dénommée «Parc National de Taï ». Localisé de part et d'autre du parallèle $6^{\circ}$ Nord et du méridien $7^{\circ}$ Ouest, ce parc représente à lui seul le quart de la forêt dense humide ivoirienne et les trois-quarts de la forêt hyper-ombrophile restante occupant du coup la première place des surfaces forestières sous protection de toute l'Afrique de l'Ouest. A vocation scientifique, il est inscrit depuis 1978 sur la liste du Réseau international des Réserves de la Biosphère et depuis 1982 au patrimoine mondial de l'UNESCO. Ce parc abrite environ $87 \%$ des espèces de mammifères connues de la zone forestière ouest-africaine ; soit environ 140 espèces de mammifères, près de 240 espèces d'oiseaux et plus de 1800 espèces végétales (Konaté et Kampmann, op. cit. ; Koffi, 2000).

Malheureusement, le PNT est menacé par la prolifération des localités ${ }^{2}$ dans la région du Sud-Ouest et l'accroissement de la densité de population rurale $^{3}$ (Brou, 2010; Brou et al., 2005; Ministère du Plan et du

\footnotetext{
${ }^{2}$ Plus des deux tiers des lieux habités en zone forestière se trouvent dans la région Sud-Ouest (Brou, 2010).

${ }^{3}$ Le taux de croissance démographique enregistré dans le sud-ouest est le plus élevé du pays (soit $10,9 \%$ ) et la densité de la population y est largement supérieure à la moyenne nationale (Ministère du Plan et du Développement, 2008). On dénombre 16686 campements dans cette région, pour une population totale estimée à 1395251 personnes; et on estime qu'en 2020 elle
} 
Développement, 2002). Il subit ainsi une forte pression causée par la quête de terres cultivables, les braconniers, les fabricants de charbon de bois et même les exploitants forestiers.

Pourtant, à l'instar de toute autre superficie forestière, cette aire protégée fournit de nombreux services économiques et écologiques (Sangaré, 2013). Aussi, le fait que, pour un massif forestier donné, les pertes liées à la réduction des services des écosystèmes forestiers risquent-elles d'être supérieures aux bénéfices agricoles à long terme en entraînant une aggravation de la pauvreté rurale (Poisson, 2009). En outre, d'autres intérêts multiples plaident en faveur de la restauration de cet important massif forestier. Cette dernière passe par le rétablissement du noyau central du parc et la constitution d'une aire d'adhésion dans son entourage immédiat. La mise en œuvre de cette action exige la rétrocession à l'Etat ivoirien des exploitations agricoles installées dans un certain rayon autour de ce massif forestier. Ce faisant l'analyse des conditions et des modalités particulières d'un dialogue entre l'Etat et les populations se justifie dans la mesure où la démarche du premier ne manque pas parfois de susciter de vives tensions entre les populations riveraines d'une part et entre celles-ci et les Institutions en charge des aires protégées d'autre part (Druguet, 2007 ; Wasikama, 1998).

Quoiqu'il en soit, il faudra rechercher une stratégie de développement local visant la survie du PNT tout en assurant la pérennité des activités agricoles qui reste l'un des enjeux majeurs pour l'avenir de la région. Dès lors, les questions suivantes méritent d'être posées.

Quelles sont les conditions de la restauration du PNT? En d'autres termes, est-il possible de concilier les activités agricoles et la préservation du PNT ? Faut-il absolument rétrocéder les terres agricoles avoisinantes pour restaurer le PNT ? Quels sont dans ce cas les déterminants de la rétrocession ? Quels sont en moyenne le consentement à recevoir (CAR) des paysans c'està-dire les montants qu'ils réclament par are rétrocédé ? A combien peut-on estimer le coût financier du dédommagement qu'engendrerait une éventuelle politique de restauration?

Comme hypothèse centrale, on suppose que la restauration du PNT passe absolument par une rétrocession des terres agricoles avoisinantes. Subsidiairement, on admet que le revenu, le niveau d'instruction du chef du ménage, la taille du ménage, la valeur de la production agricole, la durée de résidence dans la localité et le coût d'aménagement de la parcelle, contrairement à la sensibilité environnementale de l'enquêté, sont positivement corrélés au CAR du chef de ménage. 
Pour répondre aux questions susmentionnées, l'on s'est fixé, comme objectif général, d'analyser économiquement la restauration du PNT. De façon spécifique, il s'agit de caractériser les exploitations agricoles menaçant directement la survie du PNT; d'identifier les facteurs socio-économiques, techniques et démographiques susceptibles d'expliquer l'adhésion des populations à la politique de rétrocession des terres agricoles condition quasi sine qua non à la restauration de ce parc et d'évaluer les CAR totaux et moyens des paysans en vue de proposer une meilleure politique de dédommagement.

La section suivante dresse la revue de la littérature. La méthodologie de l'étude est décrite dans la section 3. La section 4 présente la méthode d'analyse. La section 5 analyse et discute les résultats de l'étude. La dernière section conclut l'étude.

\section{Revue de la littérature}

Bonnieux et al. (1995) ont mené une étude sur l'amélioration de la qualité des eaux littorales dans la rade de Brest en France. En se basant sur une enquête conduite auprès de 607 personnes pendant l'été 1993, ils étudient deux scénarii : le premier concerne l'amélioration de la salubrité des eaux et le second la préservation de l'écosystème menacée par une eutrophisation. Cette étude révèle que $75 \%$ des enquêtés acceptent de payer pour la salubrité et $49 \%$ pour l'écosystème. La modélisation Tobit du consentement à payer (CAP) qui est faite montre que les variables explicatives significatives sont le sexe, le revenu, le niveau d'éducation ou la profession, la sensibilité environnementale, la perception de la qualité de l'eau et la pratique d'activités récréatives. Pour finir, ils trouvent que les CAP moyens des individus sont respectivement de 215 Francs Français (soit 10\% de la facture d'eau annuelle) et 160 Francs Français respectivement pour les biens "salubrité" et "écosystème". Les auteurs soupçonnent néanmoins l'existence d'un biais d'échantillonnage dans la mesure où les personnes enquêtées peuvent ne pas être assez représentatives de la population de la rade.

Wasikama (1998) analyse la rationalité de la préservation du PNT au détriment des autres utilisations alternatives possibles des terres. Pour ce faire, il a recours à une analyse financière qui lui permet d'estimer la valeur actuelle nette maximale d'une exploitation des terres du PNT à une annuité de près de 366 milliards de Francs CFA. Puis, il mène une analyse économique qui est basée sur l'évaluation contingente et appliquée à un échantillon de 212 individus. Dans cette analyse, il procède à deux estimations économétriques : un modèle logit du CAP simple et la méthode des moindres carrées ordinaires appliquée au modèle semi-log du CAP. De ces estimations, il conclue à la nonsignificativité de l'âge dans l'explication du CAP et reste confus quant à l'influence du revenu dans l'explication du CAP compte tenu des signes contradictoires des coefficients de cette variable dans les deux estimations. 
Judez et al. (1998) ont recours à la MEC avec choix dichotomique simple pour déterminer la valeur d'usage récréatif du Parc National de Tablas de Daimiel en Espagne. Pour cela, ils se réfèrent à une technique basée sur la moyenne tronquée pour mesurer le bien-être. Leur étude atteste d'une part de l'indépendance du CAP des visiteurs d'avec l'enquêteur et la période d'enquête ; et d'autre part, de la non significativité de la distance par rapport à l'origine du visiteur, du nombre de jours passés à visiter la zone, de l'accompagnement par un ou plusieurs enfants durant la visite du parc, de l'âge, du sexe et de la fréquence annuelle de ses visites dans l'explication du CAP. Ils évaluent à environ 81,4 millions de pesetas par an la valeur récréative totale du parc, soit 943,4 pesetas la valeur moyenne d'une visite.

En Allemagne, Moon et al. (2002) ont tenté d'évaluer le CAP des Berlinois à l'égard des produits agricoles moins dégradants pour l'environnement. Ils ont pour cela constitué leur échantillon dans 6 districts de Berlin selon le niveau de revenu (faible, moyen et élevé) et le lieu d'habitation (Berlin Est et Berlin Ouest). Leur résultat révèle la non-significativité du revenu et de l'éducation dans l'estimation du CAP contrairement au lieu d'habitation. Concernant cette variable, ils trouvent que les Berlinois de l'Ouest ont un CAP plus élevé que ceux de l'Est.

Quant à Svedsäter (2003), il analyse les interprétations des populations, leurs manières de comprendre et leur sensibilité aux questions d'évaluation contingente. Il déplore le fait qu'une grande partie des enquêtés n'interprètent pas l'épreuve d'évaluation comme des intentions, et conclue à la forte sensibilité des populations aux questions d'évaluation. Pour lui, le risque d'un biais hypothétique est bien réel en évaluation contingente.

L'objectif de Kontoleon et Swanson (2003) est d'étudier la possibilité pour une "espèce charismatique" comme le Panda de la province chinoise du Sichuan de constituer des instruments pour la conservation de la nature. Tout en faisant une distinction nette entre l'espèce et son habitat, ils tentent à l'aide de la MEC d'évaluer les CAP pour la préservation du Panda et de son habitat. Trois scenarii sont utilisés : le Panda en cage, le Panda en liberté et le Panda dans une réserve. En passant du scénario de "cage" à celui de "réserve", les auteurs notent la baisse des "zéros" de 37\% à 7\%. Ils interprètent cette baisse comme une disponibilité des personnes enquêtées à améliorer les conditions de vie du Panda et atteste de ce fait l'existence de plusieurs sortes de préférences pour la conservation du Panda.

L'étude de Raboteur et Rodes (2006) porte sur les récifs coralliens dans les Caraïbes, plus précisément dans la zone de pigeon de la Guadeloupe. Leurs résultats révèlent que le sexe, le nombre de visites sur le site au cours des 12 derniers mois, la sensibilité, la situation familiale, le revenu, le nombre d'enfants et la catégorie socio-professionnelle sont, selon le modèle d'estimation (Régression simple, Modèle linéaire avec tous les zéros, Modèle 
linéaire avec les vrais zéros ou Modèle log-linéaire), les variables explicatives du CAP. Celui-ci s'élèverait en moyenne annuelle respectivement à 13,63 ; 11,$12 ; 9,5$ et à 9,82 euros selon les formes du modèle présentées ci-dessus. Cependant, les auteurs optent pour la dernière valeur découlant du modèle loglinéaire qui serait selon eux le modèle le mieux adapté à la résolution de leur problème. Ainsi, par extrapolation à des visiteurs au nombre de 24482 visiteurs par an, ils arrivent à évaluer le bénéfice social annuel à, soit 213579 euros pour un CAP moyen de 9,50 euros, soit 220773 euros pour un CAP de 9,82 euros.

Fleuret (2010) analyse les avantages liés à la restauration des milieux aquatiques de la Dives, de la Touques et de la zone entre Merville et Trouville. Il estime le CAP total des habitants entre 16,1 millions d'euros et 20,0 millions d'euros par la MEC et entre 8,4 millions d'euros et 24,2 millions d'euros par la méthode d'expérience de choix. Cette approche lui permet de comparer les deux méthodes de préférences déclarées et d'opter pour celle de l'expérience de choix. Cette méthode permet selon elle de mieux cerner la complexité des CAP malgré sa grande exigence technique. S'agissant de la MEC, elle procède à quatre estimations du modèle Tobit selon les zones considérées et une dernière pour l'ensemble de l'échantillon. Les résultats spécifiques de la MEC ont prouvé la relation positive entre le revenu et le CAP dans les cinq scénarii conduits et une relation négative entre l'âge et le CAP dans les Dives, le Littoral et dans l'ensemble.

En s'appuyant sur la MEC, Voltaire et al. (2011) mènent une analyse comparative de deux supports de paiement que sont la taxe d'hébergement et le droit d'entrée via l'étude de leurs effets sur les préférences des touristes pour la création de réserves naturelles dans le Golfe du Morbihan en France. Pour cela, ils scindent leur échantillon en deux groupes selon le support de paiement et testent l'homogénéité de ces deux groupes par le test non paramétrique de Mann-Whitney afin que les différences constatées relèvent de l'hétérogénéité des supports de paiements plutôt que des caractéristiques distinctives des groupes. Leurs résultats attestent de l'existence d'une relation négative entre l'âge de l'enquêté et le montant déclaré. Ces derniers trouvent également quelques soient les supports de paiement que le CAP augmente avec le revenu, le fait que l'enquêté habite la région parisienne ou qu'il soit de nationalité étrangère. Au terme de leur analyse, ils concluent à l'existence d'un effet significatif du mode de paiement sur le choix des individus d'accepter de payer ou non et les montants déclarés.

Madureira et al. (2011) utilisent simultanément la MEC et une technique de visualisation assistée par ordinateur pour évaluer les stratégies de gestion forestière à Coimbra au Portugal. Ils concluent au fait que les techniques de visualisation ont peu d'impact sur les évaluations énoncées par les enquêtés. Seuls les individus à niveaux d'éducation élevés réévaluaient à 
la hausse leur CAP en incorporant les informations fournies par les techniques de visualisation dans leur stratégie d'évaluation.

Carret et Loyer (2012) font une esquisse de solutions pour le financement durable du réseau d'aires protégées de Madagascar et des alternatives économiques à la déforestation d'origine agricole et énergétique. Pour cela, ils ont recours à l'évaluation monétaire des trois types de bénéfices des aires protégées (conservation de la biodiversité, éco-tourisme, protection hydrologique des bassins versants) et à l'identification des bénéficiaires (usagers de l'eau, opérateurs du tourisme, Association Nationale pour la Gestion des Aires Protégées). Ils trouvent que le CAP des usagers de l'eau (riziculteurs et consommateurs d'eau potable) en aval des bassins versants protégés par le réseau constitue théoriquement une source de financement durable (3 \$ par hectare d'aire protégée, croissant avec le temps) de celui-ci. Cette source de financement pourrait donc compenser les revenus perdus des populations empêchées de défricher les aires protégées pour cultiver du riz et récolter du bois de feu.

Lera-López et al. (2012) analysent les déterminants des CAP des populations pour la réduction des nuisances sonores et de la pollution de l'air générées par le transport routier dans les Pyrénées de Navarre en Espagne. Ils étendent le champ de leur étude à 14 localités situées à proximité des 5 principales routes de cette région dont le trafic quotidien est estimé à près de 150000 véhicules dont 30\% sont des camions. Après avoir souligné les insuffisances du tobit dans l'estimation de leur modèle, ces auteurs ont choisi d'adopter le «double hurdle model». Mais, compte tenu du fait qu'ils incorporent des variables agrégées au niveau des municipalités enquêtées capables d'entrainer des biais dans les estimations et l'inefficacité des estimateurs, ils ont recours à la méthode de correction de Moulton. Leurs résultats établissent, pour un taux d'acceptation de $60 \%$ de l'échantillon à contribuer financièrement à la réduction du bruit et de la pollution de l'air, respectivement à 8,22 euros, 9,31 euros et 9,56 euros les CAP des populations pour la réduction du bruit, ceux des personnes moyennement affectées et sérieusement affectées par la pollution de l'air. Ils concluent au fait que les jeunes, ayant un diplôme universitaire et vivant en milieu urbain expriment généralement des CAP pour la réduction du bruit plus élevés que les autres. Ils obtiennent quasiment les même résultats quant à l'explication du CAP pour la réduction de la pollution de l'air.

\section{Méthodologie de l'étude}

\section{Cadre théorique}

Le projet de restauration du PNT nécessite que soit élaboré un scénario hypothétique basé sur la MEC. Deux raisons principales plaident en faveur de 
cette méthode $^{4}$ : (1) comme la restauration du PNT n'est en lui-même qu'un projet, la démarche s'effectue naturellement de façon ex-ante ; (2) le fait que l'on n'ambitionne pas d'étudier les fonctions multi-usages du PNT, point n'est donc besoin de mener une analyse conjointe.

Dans la construction du marché hypothétique avec le PNT comme bien échangé, on suppose que l'Etat ivoirien décide de restaurer ce parc par la rétrocession des terres agricoles dans et aux alentours de ce parc. Les propriétaires de ces terres agricoles subiront ainsi une désutilité qui nécessiterait leur dédommagement.

Supposons que les riverains du parc s'identifient à un individu représentatif dont la fonction d'utilité est de la forme $U(x, z)$ où $x$ représente l'ensemble des biens marchands et $z$, la superficie de ce parc. Cet individu est supposé rationnel et guidé par la maximisation de son utilité dans le choix de la quantité consommée du bien $x$. On admet que l'étendue et la diversité biologique du PNT dépend des autorités qui sont eux-mêmes une émanation des populations bénéficiaires du service environnemental fourni par cet îlot forestier. La maximisation de l'utilité de l'individu représentatif sous la contrainte de son revenu permet de déceler une fonction de demande du bien $x$ qui se présente comme suit : $x=h(p ; z ; y)$. Ainsi, on peut déterminer une fonction d'utilité indirecte qui équivaut à :

$$
V(p ; z ; y) \equiv u[h(p ; z ; y), z]
$$

Supposons que les autorités décident de restaurer le PNT. Cette politique entrainera une désutilité à ces paysans caractérisée par le passage de l'étendue de cette superficie forestière de $z^{0}$ à $z^{1}$, alors, sans modification de prix et de revenu, on devrait naturellement s'attendre à une détérioration du bien-être de l'individu représentatif avec le passage de son niveau d'utilité de $u^{0}$ à $u^{1}$ telle que :

$$
u^{1}=v\left(p ; z^{1} ; y\right) \prec u^{0}=v\left(p ; z^{0} ; y\right)
$$

On pourrait alors s'interroger sur le montant maximum que l'individu est prêt à recevoir afin de tolérer cette détérioration de son bien-être. C'est le consentement à recevoir (CAR) qui s'identifie par l'équation suivante :

$$
v\left(p ; z^{1} ; y+C A R\right)=v\left(p ; z^{0} ; y\right)
$$

Le CAR est donc le montant que l'on pourrait ajouter au revenu de cet individu après la restauration du PNT de sorte à ce qu'il ait le même niveau d'utilité qu'il avait avant la restauration. Tel pourrait se présenter le fondement de la MEC dans le contexte de la présente étude. Ainsi, on estime l'équation suivante :

$$
\operatorname{CAR}\left(\mathrm{z}_{0} ; \mathrm{z}_{1}\right)=\mathrm{f}\left(\mathrm{p} ; \mathrm{z}_{0} ; \mathrm{z}_{1} ; Q ; \mathrm{y} ; \mathrm{T}\right)
$$

\footnotetext{
${ }^{4}$ Ces raisons ont également été avancées par Voltaire et al. (2011).
} 
avec $p$ : le vecteur de prix des biens marchands ; $z_{0}$ : la taille initiale du PNT ; $z_{1}$ : la taille finale du PNT; $Q$ : les caractéristiques des autres espaces forestiers disponibles ; $y:$ le revenu et $T:$ les caractéristiques et les goûts de l'individu représentatif.

\section{Collecte des données}

Les localités enquêtées ont été choisies parmi les sous-préfectures qui jouxtent le PNT excepté celles de Grabo et de Guiroutou du fait de leur inaccessibilité au moment de la collecte des données. Ces localités limitrophes sont potentiellement les refuges de la majorité des paysans qui squattent le parc. Elles ont été sélectionnées selon un tirage aléatoire simple et la collecte des données s'est déroulée en deux étapes. La première a permis de couvrir les localités de Taï, de Daobly et de Zagné situées sur la façade Ouest du PNT durant le mois de Septembre 2010 ; et la seconde s'est déroulée pendant le mois de Juin 2011 et elle a couvert les localités de Buyo, Konédougou, Walèbo, Kragui et Pogréagui situées sur la façade Est.

Tableau 1 : Répartition des ménages de l'échantillon selon les localités enquêtées.

\begin{tabular}{|c|c|c|c|c|}
\hline Départements & Sous-préfectures* & $\begin{array}{l}\text { Communes } \\
\text { ou Villages }\end{array}$ & $\begin{array}{c}\text { Nombre de } \\
\text { ménages enquêtés }\end{array}$ & Sous-total \\
\hline \multirow{5}{*}{ SOUBRE } & BUYO & Buyo & 131 & \multirow{5}{*}{331} \\
\hline & & Konédougou & 59 & \\
\hline & OUPOYO & Walèbo & 65 & \\
\hline & MEAGUI & Kragui & 14 & \\
\hline & & Pogréagui & 62 & \\
\hline \multirow[t]{3}{*}{ GUIGLO } & \multirow{3}{*}{ TAII } & Taï & 77 & \multirow{3}{*}{177} \\
\hline & & Daobly & 41 & \\
\hline & & Zagné & 59 & \\
\hline TOTAL & 4 & 8 & 508 & 508 \\
\hline
\end{tabular}

*4 des 6 sous-préfectures directement riveraines au PNT.

Source : construit par l'auteur.

La sélection des ménages enquêtés s'est faite par la combinaison de la méthode des pas et de celle des itinéraires. Dans les localités visitées, des districts de recensement ont été identifiés et un itinéraire a été tracé. Sur cet itinéraire, on a procédé au dénombrement puis au tirage des ménages à enquêter par la méthode des pas.

Lors de la collecte, le statut d'agriculteur des sondés est vérifié avant l'administration du questionnaire. Ce dernier est constitué de quatre sections dont la première renseigne sur les caractéristiques socio-économiques et démographiques du ménage et concerne notamment les informations relatives aux caractéristiques du chef et à la composition du ménage. Cette étape de l'enquête est conforme à l'esprit et à la lettre de toutes les études en 
évaluation contingente (Lera-López et al., 2012 ; Madureira et al., 2011 ; Voltaire et al., 2011 ; Judez et al., 1998 ; Bonnieux et al., 1995).

La seconde section a trait aux caractéristiques de l'exploitation agricole, aux relations de l'exploitant avec les différents partenaires du monde agricole, aux activités menées, ainsi que les cultures pratiquées. On y analyse également la composition de la main-d'œuvre et la durée de travail sur l'exploitation. Le but visé est de minimiser le biais de couverture qui pourrait résulter du comportement de passager clandestin de certains enquêtés. Des non agriculteurs auraient probablement des CAR sous-évalués entrainant plus tard des difficultés dans l'exécution de la politique de restauration du parc.

La troisième section, comme il est de coutume dans ce type d'étude, concerne l'évaluation de la sensibilité environnementale (Lera-López et al., 2012 ; Voltaire et al., 2011 ; Raboteur et Rodes, 2006 ; Luchini, 2002 ; Bonnieux et al., 1995) et de la sensibilité aux variations de prix agricoles de l'individu. Dans cette section, on tente d'évaluer la sensibilité de l'enquêté à l'égard de la déforestation et de son attachement aux ressources forestières. Il lui est demandé de donner son avis sur la dégradation forestière de la région, de dire à quel niveau il se sent préoccupé par ce phénomène et si son activité professionnelle bénéficie ou non de sa proximité d'avec le PNT. L'objectif est de faire prendre conscience à l'enquêté de la situation critique de cet îlot forestier. Cette démarche a été adoptée par Kontoleon et Swanson (2003) pour la préservation du Panda dans la province du Sichuan. Par ailleurs, interroger l'enquêté sur sa réaction à une variation quelconque des prix agricoles revient à tester un tant soit peu sa sensibilité environnementale.

La dernière section du questionnaire concerne l'évaluation du CAR en cas d'une éventuelle rétrocession des terres à l'Etat. Il est demandé aux enquêtés de dire s'ils sont prêts à abandonner leur terre cultivée; si oui, ils devront préciser la nature du dédommagement désiré, le nombre d'ares qu'ils seraient disposés à céder et le montant qu'ils souhaiteraient recevoir en retour.

\section{Méthode de correction des différents biais}

Dans toute étude en évaluation contingente, plusieurs biais peuvent subsister dans l'élaboration du scénario hypothétique. C'est le cas notamment du "biais hypothétique" résultant de la confrontation des personnes interrogées à un marché contingent et non à un marché réel (Bonnieux, 1998). L'existence de ce type de biais a même été prouvée par les résultats économétriques de Svedsäter (2003). Ce biais peut être résolu en spécifiant clairement le marché contingent à l'enquêté (Luchini, 2002). Il s'agit notamment de lui faire prendre conscience que l'on raisonne sur un marché fictif et non sur un marché réel sans avoir à influencer, ou le moins possible, ses réponses.

Le "biais de conception" ou "biais de couverture" résulte du fait qu'une partie des individus interrogés, n'étant pas directement impliquée par 
un format de paiement, adopte un comportement de passager clandestin, notamment en surestimant son CAP ou son CAR (Voltaire et al., 2011). L'existence de ce biais est minimisée dans cette étude par la vérification du statut d'agriculteur de l'enquêté. Le but est de se rassurer que les personnes enquêtées n'adoptent pas un comportement de passager clandestin en sousestimant leur CAR par le fait qu'elles ne soient pas agriculteurs.

Le "biais d'échantillonnage" résulte de la représentativité de 1'échantillon par rapport à la population-cible. L'individu ne se sentant pas concerné par la valorisation de l'actif naturel aura tendance à sous-estimer ou à sur-estimer son CAP ou son CAR dans la mesure où son utilité ne s'en trouvera pas directement affectée (Raboteur et Rodes, op. cit.). En enquêtant uniquement les paysans installés dans les localités limitrophes au PNT, il est fort probable que le risque lié à ce biais soit minime.

Le recours aux questions fermées et aux enchères en évaluation contingente accroit le risque de survenance du "biais d'ancrage" ou "biais du point de départ" dans la mesure où les réponses de l'enquêté en sont influencées. Ainsi, il a été décidé, à l'image de Voltaire et al. (2011) et de bien d'autres, de recourir à une question ouverte qui accorde une certaine liberté aux enquêtés dans la formulation de leur CAR ; ce qui permet d'accroitre le taux de participation à l'exercice d'évaluation.

De tous les biais qui pourraient entamer quelque peu la qualité des résultats de cette étude, le "biais stratégique" a une forte probabilité de survenance dans la mesure où les paysans pensent souvent que leurs réponses sont susceptibles d'influencer la politique envisagée. Il a donc fallu suffisamment informer les enquêtés de leur incapacité à travers leurs réponses à influencer les décisions politiques sans toutefois entrainer leur désintéressement à l'égard du scénario hypothétique.

\section{Méthode d'analyse}

\section{Analyse statistique des données d'enquête}

L'inégale répartition ${ }^{5}$ de l'échantillon de part et d'autre du PNT (Tableau 1 ci-dessus) se justifie par les pressions anthropiques d'inégales ampleurs exercées sur cet îlot forestier. En effet, dans la zone jouxtant la façade Ouest du parc, on enregistre un taux moyen de couverture forestière de l'ordre de $36,5 \%$ contre seulement $14 \%$ dans celle avoisinant la façade Est (Office Ivoirien des Parcs et Réserves, 2006).

La moyenne d'âge des personnes enquêtées est de 43 ans avec respectivement pour minimum et maximum 17 et 88 ans. L'âge médian se situe à 42 ans. La moyenne des superficies cultivées est de 9,83 hectares et la principale culture pratiquée est le cacao avec une moyenne de 12,6 hectares,

\footnotetext{
${ }^{5}$ L'échantillon est focalisé à $65,15 \%$ dans la zone jouxtant la façade Est du PNT.
} 
suivi de l'hévéa et le café. Concernant leur degré de sensibilisation environnementale, les personnes enquêtées se déclarent à $80,12 \%$ favorables à la préservation de l'environnement ; mais, ce sont $86,02 \%$ de ces personnes qui pensent que l'état devrait mettre en œuvre des politiques de préservation et de protection des espaces forestiers. Ce sont seulement 35,63\% des enquêtés qui voudraient s'adonner à la culture de bois à condition qu'il y ait des mesures d'accompagnement. Ce taux semble être acceptable et dénote de la volonté réelle des enquêtés à participer plus ou moins au "reverdissement" du pays.

Seulement 10,63\% des personnes enquêtées (soit 54 individus sur 508) se disent disposer à céder, contre un dédommagement quelconque (le plus souvent financier), une partie ou la totalité de leur superficie agricole. Ces personnes consentantes se déclarent favorables à la préservation de l'environnement à $89,8 \%$. Le pourcentage de personnes potentiellement désireuses de céder leur terre dans la "zone Ouest" $(15,25 \%$ du sous-effectif de l'Ouest) est largement supérieur à celui de la "zone Est" (6,64\% du souseffectif de l'Est). Cet état de fait pourrait s'expliquer par le phénomène de la raréfaction des espaces forestiers qui serait plus accentué dans la seconde zone par rapport à la première. Dans la "zone Ouest", les individus pensent pouvoir acquérir de nouvelles parcelles une fois qu'ils auraient céder les leurs. Alors que dans la "zone Est", les paysans pensent qu'ils ne pourront le faire aisément, et sont donc plus réticents à céder leur parcelle.

Les personnes enquêtées ont une expérience agricole moyenne de 15 ans et demi avec pour minimum 1 an et maximum 37 ans. Concernant les personnes désireuses de céder une portion ou la totalité de leur terre, on note que $74,07 \%$ d'entre elles se déclarent être beaucoup préoccupées par la déforestation dans la région du sud-ouest contre seulement 7,41\% qui en sont indifférentes. En sensibilisant donc les populations, on pourrait aisément les associer à la politique de restauration des espaces forestiers sous protection, surtout que $70,08 \%$ des personnes enquêtées reconnaissent que la forêt en général et le PNT en particulier améliore leur activité professionnelle.

Par ailleurs, les statistiques prouvent que le Sud-Ouest est une région à fort taux d'immigration. En effet, les personnes enquêtées se composent à $42,86 \%$ et $46,94 \%$ respectivement d'allochtones et d'allogènes. Aussi, le statut de secteur d'activité "refuge" reconnue à l'agriculture est-il confirmé dans la mesure où un peu moins du tiers des enquêtés (soit 30,61\%) déclarent avoir exercé un emploi précédemment à l'agriculture, c'est dire que plus des deux-tiers des enquêtés héritent de leurs plantations ou choisissent volontairement d'exercer dans l'agriculture une fois qu'ils font leur entrée dans la population active. Les principales caractéristiques des données recueillies sont décrites dans le Tableau suivant. 
Tableau 2 : Principales statistiques descriptives des données d'enquête.

\begin{tabular}{|c|c|c|c|c|}
\hline Variables & Moyenne & Minimum & Maximum & $\begin{array}{l}\text { Ecart- } \\
\text { type }\end{array}$ \\
\hline $\begin{array}{l}\text { Compensation à recevoir par are } \\
\text { concédé }\end{array}$ & 797,2349 & 0 & 100000 & 5404,45 \\
\hline Superficies cultivées (en ares) & 736,3858 & 100 & 10200 & 837,6132 \\
\hline Superficie à concéder (en ares) & 43,30709 & 0 & 2500 & 208,2483 \\
\hline $\begin{array}{l}\text { Sexe du chef de ménage }(1=\text { homme } 2 \\
=\text { femme })\end{array}$ & 1.057087 & 1 & 2 & \\
\hline Age du chef de ménage & 43,24 & 17 & 88 & 13,1724 \\
\hline Niveau d'étude du chef de ménage & 1,564961 & 1 & 3 & \\
\hline Métier secondaire & 1,643701 & 1 & 2 & \\
\hline $\begin{array}{l}\text { Exercice d'un emploi précédemment à } \\
\text { l'agriculture }\end{array}$ & 1,690945 & 1 & 2 & \\
\hline Accès au crédit $(1=$ oui $2=$ non $)$ & 1,76378 & 1 & 2 & \\
\hline $\begin{array}{l}\text { Situation matrimoniale du chef de } \\
\text { ménage }\end{array}$ & 1,11811 & 1 & 2 & \\
\hline Appartenance ethnique & 2,275591 & 1 & 3 & \\
\hline $\begin{array}{l}\text { Distance séparant l'exploitation } \\
\text { agricole du parc (en kilomètres) }\end{array}$ & 13,12795 & 0 & 60 & 12,78333 \\
\hline $\begin{array}{l}\text { Pressentiment sur la distance séparant } \\
\text { le champ du domicile }\end{array}$ & 2,198819 & 1 & 3 & \\
\hline Formation agricole $(1=$ oui $2=$ non $)$ & 1,65748 & 1 & 2 & \\
\hline $\begin{array}{l}\text { Utilisation de salarié sur l'exploitation } \\
(1=\text { oui } 2=\text { non })\end{array}$ & 1,478346 & 1 & 2 & \\
\hline Taille du ménage & 9,980315 & 1 & 45 & 6,081596 \\
\hline $\begin{array}{l}\text { Recours au groupe d'entraide }(1=\text { oui } \\
2=\text { non })\end{array}$ & 1,387795 & 1 & 2 & \\
\hline $\begin{array}{l}\text { Localisation du ménage par rapport au } \\
\text { PNT } \\
(0=\text { si zone Ouest } 1=\text { si zone Est })\end{array}$ & 0,6515748 & 0 & 1 & \\
\hline Nombre d'année d'expérience agricole & 17,3248 & 1 & 45 & 10,95674 \\
\hline $\begin{array}{l}\text { Gain mensuel des membres en dehors } \\
\text { de l'exploitation }\end{array}$ & 4294,291 & 0 & 218000 & 21559,69 \\
\hline $\begin{array}{l}\text { Montant des prises en charge dont } \\
\text { bénéficie le ménage }\end{array}$ & 3251,969 & 0 & 350000 & 23404,59 \\
\hline $\begin{array}{l}\text { Le chef de ménage souffre d'un } \\
\text { handicap } \\
(1=\text { oui } 2=\text { non })\end{array}$ & 1,127953 & 1 & 2 & \\
\hline $\begin{array}{l}\text { Présence d'au moins un malade dans le } \\
\text { ménage } \\
(1=\text { oui } 2=\text { non })\end{array}$ & 1,71063 & 1 & 2 & \\
\hline
\end{tabular}

Source : Données d'enquête. 


\section{Analyse économétrique des données d'enquête Description des variables utilisées}

\section{- $\quad$ La variable expliquée}

Le CAR du ménage est le montant minimum en Franc CFA que son chef aimerait recevoir comme dédommagement par are rétrocédé pour tolérer la désutilité de la restauration du PNT.

\section{- $\quad$ Les variables explicatives}

Ces variables se répartissent en trois grandes catégories : ce sont des variables socio-démographiques, économiques et techniques. Elles sont décrites dans le tableau suivant.

Tableau 3 : Description des variables utilisées dans l'estimation du modèle.

\begin{tabular}{|c|c|c|}
\hline VARIABLES & DESCRIPTION & NATURE \\
\hline Compense & $\begin{array}{l}\text { Consentement à recevoir moyen du ménage par are cédé } \\
\text { (en Franc CFA/are) }\end{array}$ & continue \\
\hline \multicolumn{3}{|c|}{ Facteurs sociodémographiques } \\
\hline Age & Age du chef de ménage & continue \\
\hline Sexe & Sexe du chef de ménage & discrète \\
\hline Homme & $=1$ si chef de ménage est un homme & \\
\hline Femme & $=1$ si chef de ménage est une femme & \\
\hline Nivetude & Savoir lire & discrète \\
\hline Analphabète & $=1$ si le chef de ménage est analphabète & \\
\hline Primaire & $=1$ si le chef de ménage est d'un niveau d'étude primaire & \\
\hline $\begin{array}{l}\text { Secondaire et } \\
\text { Universitaire }\end{array}$ & $\begin{array}{l}=1 \text { si le chef de ménage est d'un niveau d'étude } \\
\text { secondaire ou universitaire }\end{array}$ & \\
\hline Situmatr & Statut marital du chef de ménage & discrète \\
\hline Marié & $=1$ si le chef de ménage vit avec une femme & \\
\hline Non marié & $=1$ si le chef de ménage est un célibataire. & \\
\hline emploi & Exercice d'un emploi précédent à l'agriculture & discrète \\
\hline Oui & $=1$ si le chef a exercé un emploi précédemment & \\
\hline Non & $=1$ si l'agriculture est son premier emploi & \\
\hline eth & Groupe ethnique du chef de ménage & discrète \\
\hline Autochtone & $=1$ si le chef est originaire de la région & \\
\hline Allochtone & $=1$ si le chef est ivoirien mais non originaire & \\
\hline Allogène & $=1$ si le chef est d'origine étrangère & \\
\hline Taille du ménage & Nombre de personnes vivant dans le ménage. & continue \\
\hline \multicolumn{3}{|c|}{ Facteurs économiques } \\
\hline $\begin{array}{l}\text { Recetteagri } \\
\text { coutpar }\end{array}$ & $\begin{array}{l}\text { Valeur de la dernière récolte réalisée sur l'exploitation } \\
\text { Coût engagé pour l'aménagement de la parcelle cultivée }\end{array}$ & $\begin{array}{l}\text { continue } \\
\text { continue }\end{array}$ \\
\hline gain & $\begin{array}{l}\text { Gain mensuel des membres exerçant hors de } \\
\text { l'exploitation }\end{array}$ & continue \\
\hline Montan & Le ménage bénéficie de prises en charge & discrète \\
\hline Oui & $=1$ si le ménage perçoit une prise en charge & \\
\hline Non & $=1$ si le ménage ne perçoit rien & \\
\hline \multicolumn{3}{|l|}{ Facteurs techniques } \\
\hline aneprai & Nombre d'années d'expérience en agriculture & continue \\
\hline Fav_env & Propension du chef à préserver l'environnement & discrète \\
\hline Oui & $=1$ si le chef est favorable & \\
\hline Non & $=1$ si le chef n'est pas favorable & \\
\hline
\end{tabular}




\begin{tabular}{lll} 
Crédit & Accès au crédit agricole & discrète \\
Oui & $=1$ si le chef a accès au crédit agricole & \\
Non & 1 si le chef n'a pas accès au crédit agricole & \\
formagri & Bénéficiaire d'une formation agricole & discrète \\
Oui & $=1$ si le chef a bénéficié d'une formation agricole & \\
Non & $=1$ si le chef n'a pas eu de formation agricole & \\
propchp & Propriété de la parcelle cultivée & discrète \\
Oui & $=1$ si le chef est propriétaire de la parcelle & \\
Non & $=1$ si le chef n'est pas propriétaire & \\
handicap & Etat de santé du chef de ménage & continue \\
Oui & $=1$ si le chef de ménage souffre d'un handicap & \\
Non & $=1$ si le chef de ménage ne souffre d'aucun handicap & \\
\hline \hline A suivre (page suivante). & \\
& & \\
Tableau 3 (suite). & & NATURE \\
\hline \hline VARIABLES & DESCRIPTION & discrète \\
\hline handmen & Présence d'un malade au sein du ménage & \\
Oui & $=1$ si le ménage a en son sein un malade & \\
Non & $=1$ si le ménage n'a pas de malade en son sein & \\
Site & Localisation par rapport au PNT & \\
Façade ouest & $=1$ si le ménage est installé dur la façade Ouest du PNT & \\
Façade est & $=1$ si le ménage est installé dur la façade Est du PNT & \\
$\begin{array}{l}\text { Distchp } \\
\text { Très éloigné }\end{array}$ & Distance séparant l'exploitation du domicile & discrète \\
$\begin{array}{l}\text { Eloigné } \\
\text { proche }\end{array}$ & $=1$ si le chef pense que son champ est très éloigné & \\
Disparc & $=1$ si le chef pense que son champ est éloigné & \\
\hline \hline
\end{tabular}

Source : construit par l'auteur.

\section{Justification et Effets attendus des variables explicatives utilisées} variables :

La justification et les effets attendus sont exposés par catégories de - Les variables socioéconomiques et démographiques

- $\quad$ Les superficies cultivées

De l'importance des superficies possédées dépendra la superficie cédée et le montant exigé en retour ; on peut donc prédire une relation positive entre ces superficies et le CAR des paysans.

- $\quad$ La production agricole en valeur

Cette variable peut naturellement approximer le niveau de vie en milieu rural et pourrait donc évidemment influencer de manière positive le CAR du paysan. 
- $\quad$ Age du chef de ménage

Plus l'individu est âgé, moins il est productif ; il sera alors plus enclin à céder sa parcelle et à exiger un montant plus élevé comme dédommagement. - $\quad$ Sexe du chef de ménage

Les hommes devraient logiquement déclarer un CAR plus élevé que les femmes en raison de leur disposition naturelle à être des chefs de ménage. - $\quad$ Exercice d'une activité secondaire

L'individu qui exerce une activité secondaire exprimera certainement un CAR moins élevé comparativement à un agriculteur à plein temps car disposant d'une autre source de revenu.

- $\quad$ Exercice d'un emploi précédemment à l'agriculture

Un individu transfuge d'un autre secteur d'activité devrait éventuellement accorder moins de valeur à son exploitation et exprimer un faible CAR comparativement aux individus ayant toujours exercé dans l'agriculture.

- $\quad$ Niveau d'instruction du chef de ménage

L'instruction améliore la compréhension du fonctionnement $\mathrm{du}$ marché hypothétique et accroit de ce fait les propensions de l'individu à participer au scenario et à réduire son CAR.

- Accès au crédit

L'accès au crédit est une variable essentielle dans l'explication de la productivité et de la production agricole. De ce fait, il pourrait impacter négativement sur son CAR.

- $\quad$ Statut matrimonial

Les mariés pourraient logiquement être moins enclins à céder leur terre et donc exigeraient un CAR plus élevé par rapport aux autres.

- Appartenance ethnique

L'origine ethnique de l'individu peut éventuellement expliquer son CAR dans la mesure où, s'il est autochtone de la région, il pourrait exprimer un CAR un peu plus faible.

- $\quad$ Taille du ménage

Le chef d'un ménage de grande taille aurait tendance à réclamer un CAR plus élevé en raison de l'importance de ses charges familiales. Aussi, est-il avéré que la présence d'enfants pourrait influencer le consentement des individus (Judez et al., 1998).

- $\quad$ Gain mensuel des membres en dehors de l'exploitation

Ce gain devrait influencer négativement le CAR de son chef car ce dernier pourrait être victime d'une certaine illusion de richesse.

- $\quad$ Prises en charge dont bénéficie le ménage

Les ménages bénéficiant de prises en charge pourraient exprimer des CAR plus faibles que ceux des autres ménages non bénéficiaires. 
- $\quad$ Indice handicap du chef de ménage

En réduisant les capacités productives de l'individu, le handicap réduit les chances de celui-ci à pouvoir labourer ses parcelles et donc à céder ses parcelles à un prix (CAR) plus faible.

- $\quad$ Présence de malade au sein du ménage

Il est prouvé que la présence de malade au sein du ménage réduit son temps de travail, cela pourrait donc entrainer à la baisse le CAR du chef.

\section{- Les variables techniques}

- $\quad$ Superficie cédée

On peut en toute vraisemblance supposer l'existence d'une relation positive entre cette variable et le CAR de l'individu.

- $\quad$ Distance séparant l'exploitation du PNT

Plus l'exploitation est éloignée du parc, plus le paysan "confirme" son droit de propriété sur sa parcelle et pourrait dans ce cas logiquement réclamer un consentement plus élevé.

- $\quad$ Distance séparant le domicile de l'exploitation

Moins les exploitations sont éloignées du village, plus elles acquièrent de la valeur aux yeux de leurs propriétaires. Ainsi, ces derniers peuvent réclamer un CAR plus élevé.

- Bénéficiaire d'une formation agricole

En bénéficiant d'une formation agricole, le paysan améliore sa productivité et pourrait être moins enclin à accepter de céder sa parcelle. Ainsi, il pourrait réclamer un CAR plus élevé.

- $\quad$ Recours à la main d'œuvre salariale

Le recours à cette main-d'œuvre dénote de l'incapacité du ménage à exercer l'intégralité des activités de son exploitation ; il devrait donc impacter négativement sur le CAR du paysan.

- $\quad$ Recours aux groupes d'entraide

L'appartenance à un groupe d'entraide peut influencer négativement le CAR de l'individu dans la mesure où il pourrait juger moins fastidieux la création d'une nouvelle exploitation.

- $\quad$ Localisation par rapport au PNT

Un individu résidant dans une zone où les espaces forestiers se raréfient pourrait réclamer un CAR plus élevé du fait qu'il accorderait une valeur plus grande à son exploitation.

- $\quad$ Nombre d'années d'expérience en agriculture

L'expérience pourrait être positivement corrélée au CAR dans la mesure où l'individu estime qu'il est fortement attaché à sa parcelle et pourrait ainsi surévaluer celle-ci.

- $\quad$ Coût d'aménagement de la parcelle

Lorsque les coûts engagés pour l'aménagement de la parcelle cultivée sont importants, les individus auront tendance à surévaluer leur CAR. 
- $\quad$ La sensibilité environnementale de l'individu

Un individu à forte sensibilité environnementale devrait logiquement exprimer un faible CAR dans la mesure où il devrait pleinement adhérer à la politique envisagée.

\section{Analyse et Discussion}

Deux types de modèles à variable dépendante limitée sont généralement utilisés pour analyser le mécanisme de révélation des CAP ou CAR. Il s'agit soit d'un modèle de type tobit II (méthode en deux étapes de Heckman) quand le processus de décision est supposé séquentiel, soit d'un modèle tobit I (modèle tobit censuré simple) si ce processus est simultané (Wooldridge, 2002; Voltaire et al., 2011). S'il est vrai que les résultats obtenus par ces deux méthodes peuvent être sensiblement différents (N'Guessan, 2008), le choix entre ces méthodes peut se faire suite à un test d'existence d'un biais de sélection. Ce test repose sur la significativité du coefficient de l'inverse du ratio de Mill (Wooldridge, 2002). La non significativité de ce coefficient (voir Tableau 4 ci-dessous) atteste d'une part l'indépendance entre les parties qualitative et quantitative du modèle et confirme d'autre part que l'échantillon composé de personnes consentantes à la politique de rétrocession peuvent du point de vue statistique valablement représentée l'échantillon global. Ainsi, il a été décidé d'estimer un tobit censuré simple.

Mais avant de poursuivre l'analyse, le faible taux des personnes désireuses de rétrocéder leur terre plaide en faveur de la détection des variables décisionnelles de la participation ou non des personnes enquêtées au processus de rétrocession. Ainsi, dans l'équation décisionnelle, on constate que les personnes qui sont propriétaires de leurs parcelles ou qui se déclarent favorables à la préservation de l'environnement, de même que les personnes non-autochtones (allochtones et allogènes) ont une forte propension à rétrocéder leurs parcelles cultivées (Tableau A.1).

L'estimation du tobit censuré simple est précédée par les tests de Ramsey Reset et du VIF : le premier test a prouvé la bonne spécification du modèle (Tableau A.2) ; quant au second, il confirme bien l'absence de multicollinéarité entre les variables explicatives avec un VIF moyen de 1,61 inférieur à 2 (Tableau A.3). Les meilleurs résultats d'estimation du modèle empirique ayant été obtenus sous la forme log-log, on a donc naturellement choisi d'analyser ces résultats (Tableau 4 ci-dessous). 
Tableau 4 : Résultats d'estimation du Tobit censuré simple.

\begin{tabular}{|c|c|c|}
\hline VARIABLES EXPLICATIVES & COEFFICIENT & P-VALUE \\
\hline Superficies cultivées & 1,868186 & 0,165 \\
\hline Valeur de la production agricole &,- 504013 & 0,537 \\
\hline Superficie à céder & 4,378885 & $0.000^{* * * *}$ \\
\hline Age du chef de ménage & $-6,087327$ & $0,082^{*}$ \\
\hline \multicolumn{3}{|l|}{ Sexe du chef de ménage } \\
\hline Masculin & -- & -- \\
\hline Féminin & 1,665919 & 0,734 \\
\hline \multicolumn{3}{|l|}{ Exercice d'un métier secondaire } \\
\hline Oui & -- & -- \\
\hline Non & $-3,9659$ & $0,026^{* *}$ \\
\hline \multicolumn{3}{|c|}{ Exercice d'un emploi précédemment à l'agriculture } \\
\hline Oui & -- & -- \\
\hline Non & 3,858469 & $0,040^{* *}$ \\
\hline \multicolumn{3}{|l|}{ Niveau d'instruction du chef de ménage } \\
\hline Analphabète & -- & -- \\
\hline Primaire & 3,896241 & $0,074^{*}$ \\
\hline Secondaire et Universitaire & $-8,446437$ & $0,006^{* * *}$ \\
\hline \multicolumn{3}{|l|}{ Accès au crédit } \\
\hline Oui & -- & -- \\
\hline Non & $-0,8110498$ & 0,682 \\
\hline \multicolumn{3}{|l|}{$\begin{array}{l}\text { A suivre (page suivante). } \\
\text { Tableau } 4 \text { (suite). }\end{array}$} \\
\hline VARIABLES EXPLICATIVES & COEFFICIENT & P-VALUE \\
\hline \multicolumn{3}{|l|}{ Situation matrimoniale du chef de ménage } \\
\hline Marié & -- & -- \\
\hline Non marié & $-0,4080709$ & 0,878 \\
\hline \multicolumn{3}{|l|}{ Groupe ethnique } \\
\hline Autochtone & -- & -- \\
\hline Allochtone & 6,3059 & 0,117 \\
\hline Allogène & 4,965951 & 0,210 \\
\hline Distance entre l'exploitation et le PNT & $-0,2886294$ & 0,653 \\
\hline \multicolumn{3}{|l|}{ Distance champ } \\
\hline Très éloigné & -- & -- \\
\hline Eloigné & 4,203712 & $0,032^{* *}$ \\
\hline Proche & 4,676948 & $0,022^{* *}$ \\
\hline \multicolumn{3}{|l|}{ Ayant bénéficié d'une formation agricole } \\
\hline Oui & -- & -- \\
\hline Non & $-0,4863169$ & 0,751 \\
\hline \multicolumn{3}{|l|}{ Recours à la main d'œuvre salariale } \\
\hline Oui & -- & -- \\
\hline Non & 3,142951 & 0,176 \\
\hline Taille du ménage & $-2,216394$ & 0,100 \\
\hline \multicolumn{3}{|l|}{ Recours aux groupes d'entraide } \\
\hline Oui & -- & -- \\
\hline Non & $-4,939999$ & $0,010^{* *}$ \\
\hline
\end{tabular}


Façade ouest

Façade est

$-$

2,255499

$-1,131883$

0,2582946

0,2024864

$-0,0078152$

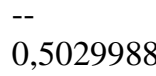

Non

Présence d'au moins un malade au sein du ménage

Oui

Non

Individu favorable à la préservation de l'environnement

Oui

Non

Constante
$--$

1,502388

$--$

$-5,156943$

$-6,060948$
0,788

0,358

0,322

0,202

0,389

0,981

$--$

(

0,455

\begin{tabular}{lcc}
\hline Rho & 0.04 & 0.841 \\
\hline
\end{tabular}

Nombre d'observations $=508 \quad$ LR chi2 $(28)=260.74$

Log likelihood $=-135.53353 \quad$ Pseudo R2 $=0.4903$

***significatif à $1 \% \quad * *$ significatif à $5 \% \quad *$ significatif à $10 \%$

Source : calculs de l'auteur.

Ces résultats montrent bien la significativité globale du modèle (Prob $>$ chi2 $=0.0000)$. Et les variables explicatives significatives se répartissent en trois grandes catégories :

Comme variables sociodémographiques, on a l'âge du chef de ménage, l'exercice d'un métier secondaire et d'un emploi précédemment à l'agriculture, le niveau d'étude de celui-ci et le recours du ménage aux groupes d'entraide. Lorsque l'âge du chef de ménage augmente d'une unité, son CAR diminue de 6,08\%. Avec le temps, les superficies cultivées perdent de leur valeur en raison de la diminution des capacités de production de leur propriétaire ; ces derniers sont donc naturellement amenés à revoir à la baisse leur CAR. Aussi, les personnes exerçant à temps partiel en agriculture ontelles en moyenne un CAR inférieur de 3,96\% à celui des personnes y exerçant à plein temps. L'existence de source de revenu en dehors du secteur agricole conduit les exploitants à diminuer leur CAR. Egalement les personnes qui n'ont jamais exercé en dehors de l'agriculture ont des CAR supérieurs de $3,86 \%$ à ceux des personnes transfuges dans le secteur. Le fait que ces personnes ne connaissent aucune autre activité excepté l'agriculture entraine une certaine surévaluation de leur exploitation et implicitement de leur CAR. Les personnes de niveau d'instruction primaire ont en moyenne un CAR supérieur de 3,89\% à ceux des analphabètes ; alors que celles qui ont un niveau d'instruction secondaire et/ou universitaire ont un CAR inférieur de 8,45\% toujours par rapport à ceux des analphabètes. Si logiquement on peut admettre que les personnes à niveau d'éducation primaire accordent plus de valeur à 
leur exploitation, on peut également supposer que les personnes à niveau d'instruction secondaire et/ou universitaire sont moins attachées à leur terre. Ils considèrent l'activité agricole comme une solution palliative en attendant qu'ils aient des opportunités d'emploi dans d'autres secteurs d'activité. Les personnes qui n'ont pas recours aux groupes d'entraide ont des CAR inférieurs de 4,94\% à ceux des chefs de ménage qui en ont recours. On pourrait alors supposer que ces personnes disposent de main d'œuvre suffisante pour subvenir à leurs besoins. Ainsi, ces chefs de ménage énoncent des CAR faibles relativement faibles.

Dans la catégorie des variables économiques, ni la valeur de la production réalisée par l'enquêté ni le coût d'aménagement initial de la parcelle, encore moins le gain obtenu par les membres du ménage en dehors de l'exploitation familiale et le montant des prises en charge dont bénéficie le ménage n'expliquent significativement le CAR des paysans.

Dans la catégorie des variables techniques, ce sont les superficies susceptibles d'être cédées, la distance séparant 1'exploitation du ménage et la sensibilité environnementale du chef de ménage qui expliquent le CAR des enquêtés. Naturellement, plus importante est la superficie que le chef de ménage veut céder, plus élevée est le montant qu'il désirerait recevoir en contrepartie. Pour une augmentation d'un are de cette superficie, le CAR de l'enquêté augmente de 4,38\%. Pour finir, les personnes qui soutiennent que leurs exploitations sont proches de leur domicile expriment un CAR moyen de $4,68 \%$ supérieur à ceux des personnes qui prétendent avoir des exploitations très éloignées. Toujours par rapport à ces derniers, les ménages qui considèrent que leur exploitation est éloignée de leur domicile ont un consentement supérieur de 4,20\%. Cela s'explique par le fait que les individus accordent plus de valeur aux exploitations proches du village.

Contrairement à Bonnieux et al. (1995) et Voltaire et al. (2011) qui établissent une relation positive entre le revenu et les CAP des enquêtés et à Judez et al. (1998) qui trouvent plutôt une relation négative entre ceux-ci, cette étude conclut à la non-significativité du revenu, approximé par la valeur de la production réalisée, dans l'explication du CAR des individus. Ce résultat vient en quelque sorte confirmé celui de Moon et al. (2002). Toutefois, contrairement à ces derniers, la significativité du niveau d'instruction dans l'explication du CAR est prouvée. Le passage de l'état d'analphabète à celui d'individu à niveau d'instruction primaire emmène le concerné à augmenter son CAR. Ce résultat confirme ainsi partiellement les conclusions de Madureira et al. (2011) et de Bonnieux et al. (1995). Ces conclusions sont rapidement infirmées lorsque l'individu passe de la situation d'analphabète à celle d'individu à niveau d'instruction secondaire ou universitaire. En effet, une telle évolution emmène l'individu enquêté à réduire son CAR. 
A l'image de Judez et al. (1998), cette étude atteste la non significativité de la distance dans l'explication du CAR des individus ; apportant ainsi une infirmation au résultat de Moon et al (2002) quant à la contribution du lieu d'habitation dans l'explication du CAP.

Alors que Voltaire et al. (2011) estiment que les personnes étrangères expriment un CAP supérieur à celui des nationaux français quant à la préservation des réserves naturelles du Golfe de Morbihan, cette étude montre que l'origine ethnique n'explique aucunement le CAR des paysans. A priori, on ne peut donc faire aucune distinction entre les autochtones, les allochtones et les allogènes quant à leur adhésion à la politique de restauration du PNT.

Cette étude corrobore les résultats de Voltaire et al. (op. cit.) et de Raboteur et Rodes (2006) quant à la non significativité du sexe du chef de ménage dans l'explication du consentement des enquêtés. Tout comme LeraLópez et al. (2012), mais contrairement à Wasikama (1998), la contribution significative de l'âge dans l'explication du CAR des individus est démontrée.

De manière tout à fait logique, l'on constate que le CAR est à la hauteur des sacrifices consentis. Plus importante est la superficie cédée, plus élevé est le CAR de l'individu.

Des variables, que l'on a fortement soupçonnés au départ, se sont finalement avérés non significatives. Il s'agit particulièrement de la valeur de la production réalisée sur la parcelle cultivée, l'expérience agricole de l'individu, le coût d'aménagement initial de la parcelle cultivée, le gain mensuel des membres du ménage en dehors de l'exploitation et le montant des prises en charge dont bénéficie le ménage.

\section{Conclusion}

L'objectif de cette étude était de discuter de la faisabilité pratique d'une politique de restauration du PNT via la rétrocession des parcelles agricoles dans l'aire d'adhésion de ce massif forestier. Pour cela, la MEC a été utilisée pour analyser l'acceptation du projet par les paysans et la mise en œuvre pratique d'un tel projet. Les résultats ont permis de faire un certain nombre d'observations.

Dans un premier temps, on démontre que l'accroissement démographique dans la région du Sud-Ouest, avec son corollaire de demande accrue de terres agricoles, exerce une forte pression sur les massifs forestiers de la région et particulièrement ceux avoisinant le PNT. La restauration de ce parc passe donc inéluctablement par une expropriation des paysans installés dans l'aire d'adhésion de ce parc.

Dans un deuxième temps, il a été prouvé que, quoique difficile à mettre en œuvre vu le taux assez faible de personnes désireuses de céder volontairement leur parcelle agricole (seulement $10,63 \%$ des personnes enquêtées), l'expropriation reste possible dans la mesure où près du quart de 
ces personnes qui adhérent au projet de restauration ne réclament pas de dédommagement en cas de rétrocession de leurs parcelles cultivées. Comme quoi, elles reconnaitraient l'implantation illégale de leurs exploitations agricoles dans l'aire d'adhésion du PNT. Toutefois, cette expropriation nécessitera qu'un fonds substantiel soit dégagé pour dédommager les paysans déguerpis. Notons que trois personnes sur quatre parmi celles qui désirent céder leur terre réclament en contrepartie un dédommagement. C'est ce qui a présidé à l'analyse du CAR de ces derniers en guise de dédommagement. La restauration du PNT reste tout à fait plausible dans la mesure où un pourcentage assez substantiel (environ 35\%) de l'échantillon désire s'investir dans la culture du bois de teck à condition que certaines facilités leur soient accordées.

Pour finir, les résultats de l'étude attestent la contribution des facteurs tels que le niveau d'instruction, la pratique de l'agriculture à temps partiel, l'appartenance à un groupe d'entraide, l'exercice d'un emploi précédemment à l'agriculture, les superficies cédées, le rapprochement de l'exploitation avec le lieu d'habitation et surtout la sensibilité environnementale de l'individu dans l'explication simultanée de la décision de cession des parcelles détenues et des montants réclamés en guise de dédommagement.

\section{Implications de politiques économiques et environnementales}

$\mathrm{Au}$ regard de la significativité du coefficient de la sensibilité environnementale de l'individu et de l'adhésion d'une frange non négligeable de la population enquêtée à la politique de reboisement, il urge de procéder à la sensibilisation des populations surtout riveraines à la sauvegarde des massifs forestiers de la région du Sud-Ouest et particulièrement celle du PNT. En cela, les différentes associations (culturelles, religieuses, etc.) auxquelles appartiennent la majorité des paysans pourraient servir de canaux de transmission. Aussi, convient-il dans la campagne de sensibilisation d'accroitre la participation des populations à la politique de rétrocession en ciblant principalement les individus à forte sensibilité environnementale, propriétaires de leur parcelle et étant non-autochtones à la région du SudOuest.

Par ailleurs, il faut promouvoir les opportunités d'emplois nonagricoles dans cette région en ciblant particulièrement les jeunes. En effet, en offrant plus d'emplois dans le secteur non agricole aux jeunes, l'on pourra détourner cette frange de la population des travaux champêtres et accroitre leur adhésion à la politique de reforestation. En effet, il est prouvé que les jeunes ont des CAR plus élevés par rapport aux personnes âgées. En outre, l'intensification du système agricole (adoption de variétés plus productives) permettra de réduire la consommation de superficies forestières et de freiner les menaces contre le PNT. 
Pour finir, il faut réfléchir à la mise en œuvre d'une politique de reboisement associant les populations rurales et d'un système de cogestion des massifs forestiers dont les riverains seront évidemment les premiers bénéficiaires.

\section{References:}

1. Babu S. C. and Hassan R. (1995) International Migration and Environmental Degradation - The Case of Mozambican Refugees and Forest Resources in Malawi, Journal of Environmental Management 43, 223-247.

2. Bonnieux F. (1998) Principes, mise en œuvre et limites de la méthode d'évaluation contingente, INRA-Economie, Rennes.

3. Bonnieux F., Le Goffe P. et Vermersch D. (1995) La méthode d'évaluation contingente : application à la qualité des eaux littorales, in: Économie \& prévision. Numéro 117-118, 1995-1-2. Agriculture et environnement. 89-106.

4. Brou T. (2010) Variabilité climatique, déforestation et dynamique agrodémographique en Côte d'Ivoire, Sécheresse, 21 (1e), 1-6.

5. Brou Y. T., Oszwald J., Bigot S. et Servat E. (2005) Risques de déforestation dans le domaine permanent de l'Etat en Côte d'Ivoire : Quel avenir pour ces derniers massifs forestiers ?, Télédétection, vol. $5, \mathrm{n}^{\circ}(1-2-3), 105-121$.

6. Carret J.-C. et Loyer D. (2012) Comment financer durablement le réseau d'aires protégées terrestres à Madagascar? Apport de l'analyse économique, World Parks Congress/Durban, Workshop "Building comprehensive protected areas systems". consulté le 18 Janvier 2012 à l'adresse :

7. http://iwlearn.net/abt_iwlearn/events/ouagadougou/readingfiles/world bank-comment-financer.pdf

8. Champ P. A., Flores N. E., Brown T. C. et Chivers J. (2002) Contingent Valuation and Incentives, Land Economics, vol. 78, $\mathrm{n}^{\circ} 4$, 591-604.

9. Coulibaly N. (1998) Déforestation et activités agricoles en Côte d'Ivoire: Recherche d'un nouvel équilibre. Thèse de Doctorat Université de Laval Québec.

10. Desaigues B. et Point P. (1990) Les méthodes de détermination d'indicateurs de valeur ayant la dimension de prix pour les composantes du patrimoine naturel, Revue économique, Vol. 41, №2, Économie de l'environnement et du patrimoine naturel, 269-319.

11. Druguet A. (2007) Concilier agriculture et conservation d'un paysage de terrasses à la périphérie du Parc national des Cévennes, 
Géocarrefour, vol. 82/4 | 2007, mis en ligne le 31 décembre 2010. URL : http://geocarrefour.revues.org/3092

12. Fleuret A. (2010) Les avantages liés à la restauration de quatre milieux aquatiques voisins, Etudes et Documents $\mathrm{n}^{\circ} 28$.

13. Héritier S. (2007) Les parcs nationaux entre conservation durable et développement local, Géocarrefour, Vol. 82/4, mis en ligne le 06 juin 2008. URL : http://geocarrefour.revues.org/2992

14. Júdez L., de Andrés R., Hugalde C. P., Urzainqui E. and Ibáñez M. (1998) Évaluation contingente de l'usage récréatif d'une réserve naturelle humide, Cahiers d'économie et sociologie rurales, $\mathrm{n}^{\circ} 48$.

15. Koffi N. (2000) Présentation du Parc National de Taï, in Etat des recherches en cours dans le Parc National de Taï (PNT), Rapport du Séminaire du 8 au 10 mars 2000, sous la Direction de Girardin, O. ; I. Koné et $\mathrm{Y}$. Tano

16. Konaté, S. et D. Kampmann (2010), Atlas de la Biodiversité de l'Afrique de l'Ouest, Volume III, Côte d'Ivoire, Germany.

17. Kontoleon A. et Swanson T. (2003) The Willingness to Pay for Property Rights for the Giant Panda: Can a Charismatic Species Be an Instrument for Nature Conservation?, Land Economics, vol. 79, $\mathrm{n}^{\circ} 4$, 483-499.

18. Lera-López F., Faulin J. and Sánchez M. (2012) Determinants of the willingness-to-pay for reducing the environmental impacts of road transportation, Transportation Research Part D 17, 215-220.

19. Lescuyer G. (2000) Evaluation économique et gestion viable de la forêt tropicale : Réflexion sur un mode de coordination des usages d'une forêt de l'Est-Cameroun, Thèse pour l'obtention du titre de Docteur de l'E.H.E.S.S.

20. Luchini S. (2002) De la singularité de la méthode d'évaluation contingente", Economie et Statistique, n ${ }^{\circ} 357-358,141-152$.

21. Madureira L., Nunes L. C., Borges J. G. and Falcão A. O. (2011) Assessing forest management strategies using a contingent valuation approach and advanced visualisation techniques: A Portuguese case study, Journal of Forest Economics, vol. 17, 399-414.

22. Ministère de l'Agriculture et des Ressources Animales (2007) Annuaire des Statistiques Agricoles 2005, Direction des Statistiques, de la Documentation et de l'Informatique, Ministère de l'Agriculture et des Ressources Animales.

23. Ministère du Plan et du Développement (2012) Plan National de Développement 2012-2015, République de Côte d'Ivoire.

24. Ministère du Plan et du Développement (2008) Document de Stratégie pour la Réduction de la Pauvreté 2009-2013, Abidjan. 
25. Ministère du Plan et du Développement (2002) Document de Stratégie pour la Réduction de la Pauvreté, Cabinet du Premier Ministre Ministère de la Planification du Développement. République de Côte d'Ivoire.

26. Moon W., Florkowski W. J., Brückner B. and Schonhof I. (2002) Willingness to Pay for Environmental Practices: Implications for EcoLabeling, Land Economics, vol. 78, $\mathrm{n}^{\circ} 1.88-102$.

27. N'Guessan C. F. J. (2008) Le consentement des ménages ruraux à payer une prime d'assurance maladie en Côte d'Ivoire, Revue d'économie du développement 22, 101-124.

28. Niesten E. et Rice R. (2004) Gestion durable des forêts et incitations directes à la conservation de la biodiversité", Tiers-Monde. tome 45, $\mathrm{n}^{\circ} 177.129-152$.

29. N'Kongon Y. J. (2011) Evaluation de l'autofinancement de l'extension du réseau de distribution d'eau potable dans les quartiers précaires du District d'Abidjan, Final Report, AERC, Nairobi, Kenya.

30. Office Ivoirien des Parcs et Réserves (2006) Plan d'aménagement et de gestion du Parc national de Taï, Office Ivoirien des Parcs et Réserves, l'environnement, Ministère des Eaux et Forêts.

31. Poisson J. (2009) Impact de la gestion participative sur l'efficacité de conservation dans les parcs nationaux des pays sous-développés, Faculté des Sciences Université de Sherbrooke, Québec, Canada

32. Raboteur J. et Rodes M.-F. (2006) Application de la Méthode d'Evaluation Contingente aux récifs coralliens dans la Caraïbe : Etude appliquée à la zone de Pigeon de la Guadeloupe, La Revue électronique en Sciences de l'Environnement VertigO, vol. 7, $\mathrm{n}^{\circ} 1$.

33. Sangaré M. (2013) Déforestation et Agriculture en milieu rural ivoirien, Thèse unique, UFR-SEG, Université de Cocody-Abidjan.

34. Sangaré M. (2012) Effets de la raréfaction des espaces forestiers sur les niveaux de productions agricoles, Papier en cours d'instruction dans une revue scientifique internationale.

35. Shechter M. (2000) Valuing the environment, in Folmer, H. and H. L. Gabel (2000), Principles of Environmental and Resource Economics, New Horizons in Environmental Economics, Second Edition, 72-103.

36. Svedsäter, H. (2003) Economic Valuation of the Environment: How Citizens Make Sense of Contingent Valuation Questions, Land Economics, vol. 79, $\mathrm{n}^{\circ} 1,122-135$.

37. Voltaire L., Nassiri A., Bailly D. et Boncoeur J. (2011) Effet d'une taxe et d'un droit d'entrée sur les consentements à payer des touristes pour de nouvelles réserves naturelles dans le Golfe du Morbihan, Revue d'Etudes en Agriculture et Environnement, 92 (2), 183-209. 
38. Wasikama T. M. C. (1998) Utilisation alternative des terres: une analyse économique de la préservation des forêts tropicales primaires (cas du Parc National de TAÏ, SUD-OUEST de la Côte d'Ivoire). Thèse de Doctorat, Université Nationale de Côte d'Ivoire, CIRES.

39. Wooldridge J. M. (2002) Econometric Analysis of Cross Section and Panel Data, Massachusetts Institute of Technology, 741 pages.

\section{Annexes}

Tableau A.1: Résultats d'estimation du modèle décisionnel

\begin{tabular}{|c|c|c|}
\hline \multirow{2}{*}{$\begin{array}{cc}* * * \text { significatif à } 1 \% & * * \text { significatif à } 5 \% \\
\text { VARIABLES EXPLICATIVES } & \end{array}$} & \multicolumn{2}{|c|}{ * significatif à $10 \%$} \\
\hline & COEFFICIENT & P-VALUE \\
\hline Age du chef de ménage & 0.2463344 & 0.231 \\
\hline \multicolumn{3}{|l|}{ Sexe du chef de ménage } \\
\hline Masculin & -- & -- \\
\hline Féminin & -.3737836 & 0.198 \\
\hline \multicolumn{3}{|l|}{ Exercice d'un métier secondaire } \\
\hline Oui & -- & -- \\
\hline Non & -.0439015 & 0.729 \\
\hline \multicolumn{3}{|l|}{ Propriétaire de la parcelle cultivée } \\
\hline Oui & -- & -- \\
\hline Non & -.1661753 & 0.412 \\
\hline \multicolumn{3}{|l|}{ Propriété du champ est discutée par l'Etat } \\
\hline Oui & -- & -- \\
\hline Non & -.2962588 & $0.044^{* *}$ \\
\hline \multicolumn{3}{|l|}{ Groupe ethnique } \\
\hline Autochtone & -- & -- \\
\hline Allochtone & 6579179 & $0.001^{* * *}$ \\
\hline Allogène & .8379313 & $0.000^{* * *}$ \\
\hline Taille du ménage & .0653379 & 0.505 \\
\hline Gain mensuel des membres en dehors de l'exploitation & .003706 & 0.868 \\
\hline Le montant des prises en charge dont bénéficie le ménage & .0015781 & 0.950 \\
\hline \multicolumn{3}{|l|}{ Le chef de ménage souffre-t-il un handicap } \\
\hline Oui & -- & -- \\
\hline Non & .1330111 & 0.462 \\
\hline \multicolumn{3}{|l|}{ Individu favorable à la préservation de l'environnement } \\
\hline Oui & -- & -- \\
\hline Non & -.6722309 & $0.000^{* * *}$ \\
\hline Constante & -1.609582 & 0.032 \\
\hline
\end{tabular}

Tableau A.2 : Résultats du test de Ramsey-Reset.

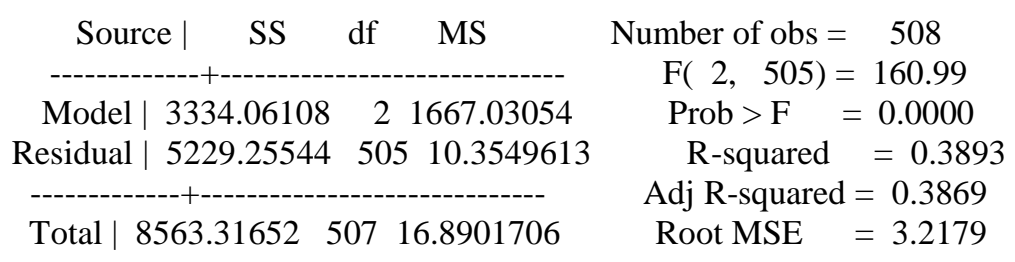




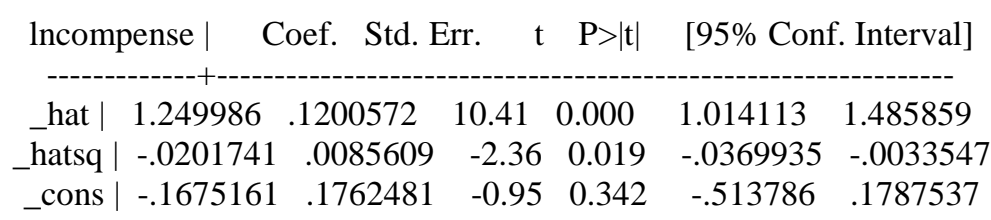

Tableau A.3 : Résultats du test de multicollinéarité.

\begin{tabular}{|c|c|c|}
\hline ble | & VIF & VIF \\
\hline _Ieth_2 & 3.58 & 9235 \\
\hline Ieth_3 & 3.54 & 0.282792 \\
\hline _Idistchp_3 & 2.33 & .428401 \\
\hline _Idistchp_2 & 2.33 & 0.429542 \\
\hline Isit & 1.94 & 0.5 \\
\hline lnaneprai & 1.87 & 0.534493 \\
\hline $\ln v$ & 1.83 & 0.5 \\
\hline lntotn & 1.56 & 567 \\
\hline lncol & 1.46 & 538 \\
\hline _Isala & 1.46 & 416 \\
\hline & 1.43 & 0.70 \\
\hline Isexe_2| & 1.41 & 0.710864 \\
\hline lnsup | & 1.41 & 0.711065 \\
\hline _Inivetude_3 & 1.39 & 0.718657 \\
\hline _Isitun & 1.37 & 7827 \\
\hline lnage | & 1.37 & 0.732425 \\
\hline _Ihandmen_2 & 1.29 & 0.77374 \\
\hline _Iemploi_2| & 1.29 & 0.773840 \\
\hline Iformagri_2 & 1.26 & 0.794335 \\
\hline Ime & 1.25 & 0.798050 \\
\hline _Inive & 1.25 & 0.799133 \\
\hline $\operatorname{lnc}$ & 1.17 & 0.85 \\
\hline Ihandicap_2 & 1.17 & 0.858041 \\
\hline lnmontan & 1.16 & 0.861015 \\
\hline Icre & 1.15 & 0.871995 \\
\hline & 1.09 & 0.916584 \\
\hline Ientraid_2 & 1.08 & 0.923918 \\
\hline & & 1.01 \\
\hline
\end{tabular}

